01

\title{
Асимптотика ядер интеграла столкновений линейного уравнения Больцмана при больших значениях индекса для потенциала твердых шаров
}

\author{
(С) Л.А. Бакалейников, Э.А. Тропп, Е.Ю. Флегонтова ฯ \\ Физико-технический институт им. А.Ф. Иоффе РАН, Санкт-Петербург, \\ Россия \\ ฯ E-mail: fl.xiees@mail.ioffe.ru
}

Поступило в Редакцию 11 мая 2018 г.

Найдена асимптотика ядер интегральных операторов, являющихся коэффициентами разложения интеграла столкновений линейного уравнения Больцмана по полиномам Лежандра, при больших значениях индекса для потенциала твердых шаров. В случае не равных по модулю скоростей взаимодействующих частиц ядра убывают экспоненциально, причем в основании экспоненты стоит отношение меньшей из скоростей к большей. В случае равных по модулю скоростей ядра убывают степенным образом.

DOI: 10.21883/PJTF.2018.16.46474.17379

Одним из методов решения линейного уравнения Больцмана является метод разложения по сферическим гармоникам. Использование такого метода сводит кинетическое уравнение к системе уравнений для коэффициентов разложения функции распределения (ФР), в которых интеграл столкновений заменяется значительно более простыми интегральными операторами, ядра которых зависят только от модулей скоростей. В существенно анизотропных задачах оказываются необходимыми учет большого числа коэффициентов разложения ФР и, следовательно, расчет ядер с большими значениями индексов. Такая ситуация возникает, например, в задаче о расчете ФР ионов. Эта задача весьма актуальна для анализа химических реакций в плазме, включающих ионы, определения подвижности ионов, нагрева нейтральной компоненты плазмы и т.д. Подходы к ее решению активно разрабатываются исследователями (см., например, обзор методов решения уравнения Больцмана для заряженных частиц [1], описание нового метода расчета 
ФР электронов и ионов в слабо ионизованной плазме [2] и ссылки в [2], сравнение результатов расчета ФР ионов с экспериментальными данными [3]).

Настоящая работа посвящена получению асимптотики ядер линейного интеграла столкновений для потенциала твердых шаров при больших значениях индекса. Отметим, что вследствие связи между линейными и нелинейными ядрами для степенных потенциалов, найденной в нашей работе [4], асимптотика линейных ядер может быть использована и для отыскания асимптотики нелинейных ядер.

Рассмотрим релаксацию малой примеси ионов с функцией распределения $f(\mathbf{v}, \mathbf{r}, t)$ на фоне основного газа с концентрацией $n_{0}$, имеющего максвелловское распределение по скоростям

$$
M_{T}(v)=\left(\frac{m}{2 \pi k T}\right)^{3 / 2} \exp \left(-\frac{m v^{2}}{2 k T}\right) .
$$

Кинетическое уравнение можно представить в виде

$$
\begin{gathered}
\left(\frac{\partial}{\partial t}+\mathbf{v} \cdot \frac{\partial}{\partial \mathbf{r}}\right) f(\mathbf{v}, \mathbf{r}, t)=-k(v) f(\mathbf{v})+\int \tilde{L}^{+}\left(\mathbf{v}, \mathbf{v}_{1}\right) f\left(\mathbf{v}_{1}\right) d \mathbf{v}_{1}, \\
k(v)=n_{0} \int\left|\mathbf{v}-\mathbf{v}_{1}\right| \Sigma M_{T}\left(v_{1}\right) d \mathbf{v}_{1},
\end{gathered}
$$

где $\Sigma=4 \pi \sigma$ - полное сечение рассеяния, $\sigma=r^{2}$ - дифференциальное сечение (в модели твердых шаров), $r$ - радиус молекулы. Выберем в качестве масштабов концентрации частиц и скорости среднюю концентрацию $n_{0}$ и тепловую скорость $v_{T}=\sqrt{2 k T / m}$ соответственно, а в качестве масштаба времени - обратную частоту столкновений $\tau_{T}=1 /\left(n_{0} \Sigma v_{T}\right)$. Введем безразмерные скорости $\mathbf{c}=\mathbf{v} / v_{T}, \mathbf{c}_{1}=\mathbf{v}_{1} / v_{T}$, безразмерную $Ф \mathrm{P} f(\mathbf{v})\left(v_{T}\right)^{3} / n_{0}$ и безразмерное ядро интеграла столкновений $L^{+}\left(\mathbf{c}, \mathbf{c}_{1}\right)=\left(v_{T}\right)^{3} \tau_{T} \tilde{L}^{+}\left(\mathbf{c} v_{T}, \mathbf{c}_{1} v_{T}\right)$. Применяя подход, использованный в [5] для расчета ядра интеграла столкновений линеаризованного уравнения Больцмана, легко получить выражение для безразмерного линейного ядра в виде

$$
\begin{aligned}
L^{+}\left(\mathbf{c}, \mathbf{c}_{1}\right) & =\frac{\exp \left(-c^{2}\right)}{2 \pi^{3 / 2}}\left|\mathbf{c}-\mathbf{c}_{1}\right| \int_{0}^{\pi} \frac{\exp \left(-\left|\mathbf{c}-\mathbf{c}_{1}\right|^{2} \operatorname{ctg}^{2}(\chi / 2)\right)}{\sin ^{4}(\chi / 2)} \\
& \times I_{0}\left(2\left|\mathbf{c} \times \mathbf{c}_{1}\right| \operatorname{ctg}(\chi / 2)\right) \sin \chi d \chi .
\end{aligned}
$$

Письма в ЖТФ, 2018, том 44, вып. 16 
Как видно из (1), ядро $L^{+}\left(\mathbf{c}, \mathbf{c}_{1}\right)$ ортогонально-инвариантно (зависит только от модулей скоростей и угла между ними), что позволяет представить его в виде разложения по полиномам Лежандра

$$
\begin{aligned}
L^{+}\left(\mathbf{c}, \mathbf{c}_{1}\right) & =\sum_{l=0}^{\infty} \frac{2 l+1}{4 \pi} L_{l}^{+}\left(c, c_{1}\right) P_{l}(\cos \theta), \quad \theta=\arccos \left(\left(\mathbf{c} \cdot \mathbf{c}_{1}\right) /\left(c c_{1}\right)\right), \\
L_{l}^{+}\left(c, c_{1}\right) & =\int_{-1}^{1} L^{+}\left(\mathbf{c}, \mathbf{c}_{1}\right) P_{l}(\cos \theta) d \Omega=2 \pi \int_{-1}^{+}\left(\mathbf{c}, \mathbf{c}_{1}\right) P_{l}(\cos \theta) \sin \theta d \theta \\
& =\frac{e^{-c^{2}}}{\sqrt{\pi}} \int_{-1}^{1} d x P_{l}(x) \sqrt{c^{2}+c_{1}^{2}-2 c c_{1} x} \\
& \times \int_{0}^{\pi} \frac{\exp \left(-\left(c^{2}+c_{1}^{2}-2 c c_{1} x\right) \operatorname{ctg}^{2}(\chi / 2)\right)}{\sin ^{4}(\chi / 2)} \\
& \times I_{0}\left(2 c c_{1} \operatorname{ctg}(\chi / 2) \sqrt{1-x^{2}}\right) \sin \chi d \chi .
\end{aligned}
$$

Будем искать асимптотику ядер $L_{l}^{+}\left(c, c_{1}\right)$. После замены переменной $q=(\sin (\chi / 2))^{-2}-1$ внутренний интеграл в (2) вычисляется аналитически [6]

$$
\begin{aligned}
2 \int_{0}^{\infty} \exp ( & \left.-\left(c^{2}+c_{1}^{2}-2 c c_{1} x\right) q\right) I_{0}\left(2 c c_{1} \sqrt{1-x^{2}} \sqrt{q}\right) d q \\
& =2 \exp \left(\frac{c^{2} c_{1}^{2}\left(1-x^{2}\right)}{\left(c^{2}+c_{1}^{2}-2 c c_{1} x\right)}\right) \frac{1}{\left(c^{2}+c_{1}^{2}-2 c c_{1} x\right)}
\end{aligned}
$$

и выражение для ядра принимает вид

$$
\begin{gathered}
L_{l}^{+}\left(c, c_{1}\right)=\frac{2 e^{-c^{2}}}{\sqrt{\pi}} U_{l}\left(c, c_{1}\right), \\
U_{l}\left(c, c_{1}\right)=\int_{-1}^{1} P_{l}(x)\left(c^{2}+c_{1}^{2}-2 c c_{1} x\right)^{-1 / 2} \exp \left(\frac{c^{2} c_{1}^{2}\left(1-x^{2}\right)}{\left(c^{2}+c_{1}^{2}-2 c c_{1} x\right)}\right) d x .
\end{gathered}
$$

3* Письма в ЖТФ, 2018, том 44, вып. 16 
Рассмотрим сначала случай $c \neq c_{1}$. Введем обозначение $a=2 c c_{1} /\left(c^{2}+\right.$ $\left.+c_{1}^{2}\right)<1$. Для получения асимптотики $U_{l}\left(c, c_{1}\right)$ применим прием, описанный в [7]. Представим множитель при полиноме Лежандра в виде интеграла Коши

$$
F(z)=\left(c^{2}+c_{1}^{2}\right)^{-1 / 2}(1-a z)^{-1 / 2} \exp \left(\frac{c c_{1} a\left(1-z^{2}\right)}{2(1-a z)}\right)=\frac{1}{2 \pi i} \oint \frac{F(t) d t}{t-z} .
$$

Интеграл здесь берется по окружности радиуса $\rho, 1<\rho<1 / a$. Используя формулу Родрига для полиномов Лежандра и интегрируя (3) $l$ раз по частям, получим

$$
U_{l}=\frac{1}{2 \pi i} \oint F(t)\left(\frac{1}{2^{l}} \int_{-1}^{1} \frac{\left(1-z^{2}\right)^{l}}{(t-z)^{l+1}} d z\right) d t=\frac{1}{2 \pi i} \oint F(t) 2 Q_{l}(t) d t .
$$

Здесь $Q_{l}(t)$ - функция Лежандра второго рода. Подставляя асимптотику $Q_{l}(t)$ при больших индексах в (4), найдем

$$
\begin{aligned}
U_{l} & =\frac{1}{2 \pi i} \frac{\sqrt{2 \pi} \Gamma(l+1)}{\Gamma(l+3 / 2)} \oint F(t) \frac{\left(t^{2}-1\right)^{-1 / 4}}{\left(\sqrt{t^{2}-1}+t\right)^{l+1 / 2}}\left(1+O\left(l^{-1}\right)\right) d t \\
& =\frac{\sqrt{2 \pi} \Gamma(l+1)}{\Gamma(l+3 / 2)} K_{l}\left(1+O\left(l^{-1}\right)\right) .
\end{aligned}
$$
Для оценки интеграла $K_{l}=\frac{1}{2 \pi i} \oint F(t) \frac{\left(t^{2}-1\right)^{-1 / 4}}{\left(\sqrt{t^{2}-1}+t\right)^{l+1 / 2}} d t$ заметим прежде
всего, что

$$
\begin{aligned}
\left(c^{2}\right. & \left.+c_{1}^{2}\right)^{1 / 2} F(t)=(1-a t)^{-1 / 2} \exp \left(\frac{a c c_{1}\left(1-t^{2}\right)}{2(1-a t)}\right) \\
& =(1-a t)^{-1 / 2} \exp \left(\frac{c c_{1}}{2(1-a t)}(a-1 / a)\right) \exp \left(\frac{c c_{1}}{2 a}(1+a t)\right)
\end{aligned}
$$

и, следовательно, точка $t=1 / a$ является трансцендентной точкой ветвления. Согласно [7], для получения асимптотики интеграла в этом случае множитель $\exp \left(c c_{1}(a-1 / a) /(2(1-a t))\right)$ следует присоединить к $\left(\sqrt{t^{2}-1}+t\right)^{-(l+1 / 2)}$ и воспользоваться методом перевала. Деформируем контур по окружности $|t|=1 / a+\eta, \eta>0$ и по петле вокруг 
разреза вдоль $[1 / a, \infty)$. Выполним замену переменной $u=-\ln a t$, выделим особенность в $\exp \left(c c_{1}(a-1 / a) /(2(1-a t))\right)$ и объединим ее c $\left(\sqrt{t^{2}-1}+t\right)^{-(l+1 / 2)}$ :

$$
\begin{aligned}
K_{l} & =\frac{\left(c^{2}+c_{1}^{2}\right)^{-1 / 2}}{a} \frac{1}{2 \pi i} \oint\left(1-e^{-u}\right)^{-1 / 2} \exp \left(-G\left(\frac{1}{1-e^{-u}}-\frac{1}{u}\right)\right) \\
& \times \exp \left(-(l+1 / 2) \ln \left(\frac{e^{-u}}{a}+\sqrt{\frac{e^{-2 u}}{a^{2}}-1}\right)-\frac{G}{u}\right) \\
& \times \exp \left(\frac{c c_{1}}{2 a}\left(1+e^{-u}\right)\right)\left(\frac{e^{-2 u}}{a^{2}}-1\right)^{-1 / 4} e^{-u} d u
\end{aligned}
$$

Здесь $G=-c c_{1}(a-1 / a) / 2>0$, интегрирование проводится по нижнему берегу разреза $[-\infty, 0)$ от $-\infty$ до 0 и по верхнему от 0 до $-\infty$. Представим показатель экспоненты, содержащей большой параметр, в виде

$$
\begin{aligned}
& -(l+1 / 2) \ln \left(\frac{e^{-u}}{a}+\sqrt{\frac{e^{-2 u}}{a^{2}}}-1\right)-\frac{G}{u} \\
& =-(l+1 / 2) \ln \left(\frac{1+\sqrt{1-a^{2}}}{a}\right)+\frac{(l+1 / 2)}{\sqrt{1-a^{2}}} f(u)-\frac{G}{f(u)}+\frac{G}{f(u)}-\frac{G}{u},
\end{aligned}
$$

где

$$
\begin{aligned}
f(u) & =-\ln \left(\left(\frac{e^{-u}}{a}+\sqrt{\frac{e^{-2 u}}{a^{2}}-1}\right) /\left(\frac{1+\sqrt{1-a^{2}}}{a}\right)\right) \sqrt{1-a^{2}} \\
& =-\ln \left(\frac{e^{-u}+\sqrt{e^{-2 u}-a^{2}}}{1+\sqrt{1-a^{2}}}\right) \sqrt{1-a^{2}} .
\end{aligned}
$$

При $u \rightarrow 0$, т. е. в окрестности особой точки, $f(u)=u+O\left(u^{2}\right)$. Сделаем в интеграле (5) замену переменной $y=f(u)$ и разложим все входящие в интеграл функции в окрестности нуля. Интеграл (5) приводится к

Письма в ЖТФ, 2018, том 44, вып. 16 
виду

$$
\begin{aligned}
K_{l} & =\frac{\left(c^{2}+c_{1}^{2}\right)^{-1 / 2}}{a} \exp \left(-\frac{G}{2}+\frac{c c_{1}}{a}-\frac{1}{2} G f^{\prime \prime}(0)\right) \\
& \times\left(\frac{1}{a^{2}}-1\right)^{-1 / 4}\left(\frac{1+\sqrt{1-a^{2}}}{a}\right)^{-(l+1 / 2)} \\
& \times \frac{1}{2 \pi i} \oint y^{-1 / 2} \sum_{k=0}^{\infty} w_{k} y^{k} \exp \left(\frac{(l+1 / 2)}{\sqrt{1-a^{2}}} y-\frac{G}{y}\right) d y, \quad w_{0}=1 .
\end{aligned}
$$

Используя подстановку $\eta=y(l+1 / 2)^{1 / 2} /\left(\left(1-a^{2}\right)^{1 / 4} G^{1 / 2}\right)$ и интегральное представление для функций Бесселя [8], найдем

$$
\begin{aligned}
& \frac{1}{2 \pi i} \oint y^{-1 / 2+k} \exp \left(\frac{(l+1 / 2)}{\sqrt{1-a^{2}}} y-\frac{G}{y}\right) d y=\left(\frac{(l+1 / 2)}{G \sqrt{1-a^{2}}}\right)^{-1 / 4-k / 2} \\
& \times \frac{1}{2 \pi i} \oint \exp \left(\sqrt{G \frac{(l+1 / 2)}{\sqrt{1-a^{2}}}}\left(\eta-\frac{1}{\eta}\right)\right) \eta^{-1 / 2+k} d \eta \\
& =\left(\frac{(l+1 / 2)}{G \sqrt{1-a^{2}}}\right)^{-1 / 4-k / 2} J_{-1 / 2-k}\left(2 \sqrt{G \frac{(l+1 / 2)}{\sqrt{1-a^{2}}}}\right) .
\end{aligned}
$$

Таким образом, разложение (6) является асимптотическим и $K_{l}$ представляется в виде

$$
\begin{aligned}
K_{l} & =\frac{\left(c^{2}+c_{1}^{2}\right)^{-1 / 2}}{\pi^{1 / 2} a} \exp \left(-\frac{G}{2}\left(1+f^{\prime \prime}(0)\right)+\frac{c c_{1}}{a}\right)\left(\frac{1}{a^{2}}-1\right)^{-1 / 4} \\
& \times\left(\frac{1+\sqrt{1-a^{2}}}{a}\right)^{-(l+1 / 2)}\left(\frac{(l+1 / 2)}{\sqrt{1-a^{2}}}\right)^{-1 / 2}\left(\cos \left(2 \sqrt{G \frac{(l+1 / 2)}{\sqrt{1-a^{2}}}}\right)\right. \\
& \left.-w_{1}\left(\frac{(l+1 / 2)}{G \sqrt{1-a^{2}}}\right)^{-1 / 2} \sin \left(2 \sqrt{G \frac{(l+1 / 2)}{\sqrt{1-a^{2}}}}\right)+O\left(l^{-1}\right)\right)
\end{aligned}
$$

Здесь учтено, что $J_{-1 / 2}(z)=\left(\frac{2}{\pi z}\right)^{1 / 2} \cos z, \quad J_{-3 / 2}(z)=-\left(\frac{2}{\pi z}\right)^{1 / 2} \times$ $\times(\sin z+\cos z / z)$. Учитывая, что $f^{\prime \prime}(0)=a^{2} /\left(1-a^{2}\right)$, окончательно 
получим

$$
\begin{aligned}
L_{l}^{+}\left(c, c_{1}\right) & =\frac{2 e^{-c^{2}} \Gamma(l+1)}{\pi^{1 / 2} \Gamma(l+3 / 2)} \exp \left(\frac{3\left(c^{2}+c_{1}^{2}\right)}{8}\right)\left(c c_{1}\right)^{-1 / 2} \\
& \times\left(\frac{\min \left(c, c_{1}\right)}{\max \left(c, c_{1}\right)}\right)^{(l+1 / 2)}(l+1 / 2)^{-1 / 2} \\
& \times\left(\cos \left(\sqrt{(l+1 / 2)\left|c^{2}-c_{1}^{2}\right|}\right)\right. \\
& \left.-w_{1} \frac{\left(c^{2}-c_{1}^{2}\right)^{2}}{2\left(c^{2}+c_{1}^{2}\right)} \frac{\sin \left(\sqrt{(l+1 / 2)\left|c^{2}-c_{1}^{2}\right|}\right)}{\sqrt{(l+1 / 2)\left|c^{2}-c_{1}^{2}\right|}}+O\left(l^{-1}\right)\right), \\
& w_{1}=-\frac{c^{2}+c_{1}^{2}}{4}\left(\frac{13}{12}+\frac{c^{2}+c_{1}^{2}+c^{2} c_{1}^{2}}{\left(c^{2}-c_{1}^{2}\right)^{2}}\right) .
\end{aligned}
$$

При совпадающих аргументах $U_{l}(c, c)$ приобретает вид

$$
U_{l}(c, c)=\frac{1}{\sqrt{2 c}} \int_{-1}^{1} P_{l}(x)(1-x)^{-1 / 2} \exp \left(\frac{c^{2}(1+x)}{2}\right) d x .
$$

Раскладывая $\exp \left(c^{2}(1+x)\right) / 2$ в ряд Тейлора в окрестности $x=1$ и пользуясь соотношением

$$
\int_{-1}^{1} P_{l}(x)(1-x)^{\alpha} d x=2^{\alpha+1} \Gamma(\alpha+1) \Gamma(l-\alpha)\left[\Gamma(-\alpha) \Gamma(l+\alpha+2]^{-1}\right.
$$

найдем

$$
L_{l}^{+}(c, c)=\frac{2}{c \sqrt{\pi}} l^{-1}\left(1+O\left(l^{-1}\right)\right) \text {. }
$$

Письма в ЖТФ, 2018, том 44, вып. 16 


\section{Список литературы}

[1] White R., Robson R., Dujko S., Nicoletopoulos P., Li B. // J. Phys. D: Appl. Phys. 2009. V. 42. N 19. P. 194001.

[2] Konovalov D.A., Cocks D.G., White R.D. // Eur. Phys. J. D. 2017. V. 71. N 10. P. 258.

[3] Mustafaev A., Grabovskiy A., Murillo O., Soukhomlinov V. // J. Phys.: Conf. Ser. 2018. V. 958. P. 012005.

[4] Эндер А.Я., Эндер И.А., Бакалейников Л.А., Флегонтова Е.Ю. // ЖТФ. 2012. T. 82. B. 6. C. $1-8$.

[5] Ферцигер Дж., Капер Г. Математическая теория процессов переноса в газах. М.: Мир, 1976. $554 \mathrm{c.}$

[6] Градштейн И.С., Рыжик И.М. Таблицы интегралов, сумм, рядов и произведений. М.: Физматгиз, 1962. $1097 \mathrm{c.}$

[7] Риекстыньи Э.Я. Асимптотические разложения интегралов. Рига: Зинатне, 1981. T. 3. $372 \mathrm{c}$.

[8] Лебедев Н.Н. Специальные функции и их приложения. М.-Л.: Физматгиз, 1963. $358 \mathrm{c}$. 